\title{
Management of Chronic Depressive Patients with Residual Symptoms
}

\author{
Changsu Han · Tzung Lieh Yeh • Masaki Kato • \\ Soichiro Sato $\cdot$ Chia-Ming Chang $\cdot$ Chi-Un Pae
}

Published online: 25 May 2013

(c) Springer International Publishing Switzerland 2013

\begin{abstract}
Various clinical issues are involved in the appropriate diagnosis and proper treatment interventions for patients with major depressive disorder (MDD). Despite a number of diverse antidepressants for treating MDD now, response and remission rates following adequate trials of antidepressant intervention are still not satisfactory. Furthermore, a significant proportion of MDD patients have residual symptoms, which are associated with increased relapse and recurrence of MDD, leading to negative impacts on the clinical course and outcomes of MDD. Timely and appropriate decision-making regarding the proper management of such cases is required in our routine daily practice. These issues are illustrated and also framed by one MDD case with a complicated clinical course. This review paper may give physicians clinical insight into how we can effectively and properly evaluate and manage such patients in clinical practice.
\end{abstract}

Previous Presentation: Part of the information presented in this article was presented at the round table discussion of psychiatrists from Asia and the USA, sponsored by Korea Otsuka International Asia and Arab.

\section{Han}

Department of Psychiatry,

Korea University College of Medicine, Seoul, Korea

T. L. Yeh

Department of Psychiatry, National Cheng Kung University Hospital, College of Medicine, National Cheng Kung University, Tainan, Taiwan

\section{Kato}

Department of Neuropsychiatry, Kansai Medical University,

Osaka, Japan

S. Sato

Zikei Hospital/Zikei Institute of Psychiatry, Okayama, Japan

\section{Introduction}

Remission is a cornerstone for complete recovery and is the standard goal of pharmacological treatment in patients with depression [1]. Residual symptoms and partial response are associated with an increased risk of relapse, faster time to relapse, a more severe and chronic course and increased functional impairment [2,3]. The importance of achieving remission has been well illustrated in the Sequenced Treatment Alternatives to Relieve Depression (STAR*D) study [2]. Remission at entry into follow-up was associated with lower relapse rates than response without remission in the STAR*D study (12-month follow-up period) [4]. Given the aforementioned findings, clinicians have to do their best to place their patients in remission as early as possible and also to maintain such a state as long as they can because the clinical meaning and importance of remission is sufficiently demonstrated in the treatment of depression. The authors discuss overall clinical aspects in diagnosis and management as well as in the use of atypical antipsychotic

\footnotetext{
C.-M. Chang

Department of Psychiatry, Chang Gung Memorial Hospital and Chang Gung University, Taoyuan, Taiwan

C.-U. Pae ( $\square)$

Department of Psychiatry, Bucheon St Mary's Hospital,

The Catholic University of Korea College of Medicine,

2 Sosa-Dong, Wonmi-Gu Bucheon, Kyounggi-Do,

420-717 Seoul, Korea

e-mail:pae@catholic.ac.kr

C.-U. Pae

Department of Psychiatry and Behavioural Sciences,

Duke University Medical Center, Durham, NC, USA
} 
(AAP) augmentation (aripiprazole) through the following case.

\section{Case Vignette: Ms C, a housewife with Residual Symptoms (Dr Han)}

Ms C, a 55-year-old homemaker and wife of a trucker, visited a outpatient clinic complaining of persistent depressed mood, palpitations, headaches and insomnia. These symptoms had started more than 5 years before her visit. She was also diagnosed as having erosive gastritis and reflux oesophagitis by her family physician, which were controlled with proton pump inhibitors, and mild glucose intolerance, which required clinical observation and lifestyle modification (i.e. diet control and exercise). A careful medical examination did not reveal any other physical problems related to her somatic symptoms (i.e. palpitations and headaches). Her family doctor had recommended that she visit a psychiatric clinic 4 years ago for control of her depression-related symptoms.

When she visited the outpatient clinic, easy forgetfulness and unexplained anxiety were also reported. She admitted to alcohol drinking and smoking habits. During her psychiatric interview, she complained of her unhappy marital life and accused her husband of 'working too hard', being 'too religious' and being 'too good a son to his mom'.

She was prescribed escitalopram, which was increased to $20 \mathrm{mg}$ a day. Additional medications given were zolpidem $(10 \mathrm{mg})$ for sleep control and lorazepam $(1.5 \mathrm{mg})$ for anxiety control. Cognitive behavioural therapy in the context of individual psychotherapy was initiated after 4 weeks of treatment. Her psychiatrist reassessed her symptoms using two forms of the Patient Health Questionnaire 9 and 15 (PHQ-9; PHQ-15) and the Beck Anxiety Inventory (BAI) after 6 weeks of treatment (HC12EESE0130).

\section{What is the Appropriate DSM-IV-TR Diagnosis of this Patient? (Dr Han)}

Ms C reported loss of pleasure and feelings of hopelessness nearly every day during the preceding 2 weeks. She also reported associated depressive symptoms, such as decreased appetite, feeling bad about herself, difficulty concentrating and thoughts of death. These symptoms lasted for more than 5 years as her index depressive episode. She could be diagnosed as having severe depression without psychotic features according to the Diagnostic and Statistical Manual of Mental Disorders, version 4 (DSMIV-TR) criteria [5]. A 'chronic' specifier could also be applied to this patient.

\section{What Would be a Useful, Optimal Way to Check Symptom Changes and Residual Symptoms of the Depressive Patients in Your Clinic? (Drs Han, Yeh, Chang, and Sato)}

In a survey study, psychiatrists' most frequent method for diagnosing depression was through a clinical interview with patients $(53 \%)$. Only $11 \%$ of psychiatrists reported that they used measurement scales [6]. The STAR*D study underscored the concept of measurement-based clinical practice, highlighting the importance of the periodic evaluation of symptoms and depression severity using measurement scales [6]. In real clinical settings, diagnosing depression is a complex and easily confounded goal because of depression's varied symptoms and comorbid medical and psychiatric conditions [7].

Depression measurement scales can be classified into self-report forms and clinician rating forms. Commonly used clinician rating depression scales are the Hamilton Depression Rating Scale (HDRS) [8] and the Montgomery Åsberg Depression Rating Scale (MADRS) [9]. The HDRS has the following advantages: emphasis on physical and vegetative symptoms; multidimensional factors; and advantages in evaluating a specific hypothesis regarding depression treatment or pathophysiology. On the other hand, the MADRS contains fewer items as compared to the HDRS, uses plain words that are easier to understand, has more items that are dependent on patients' subjective report, which allows the MADRS to be used by less experienced raters, and takes a shorter time to complete as compared with the HDRS. Furthermore, the highest score on each item is higher in the MADRS than in the HDRS, which makes the MADRS more sensitive to minor symptom changes. However, 13-15 minutes are needed to complete these clinician rating scales, and the interviewers should be trained in the proper use of a structured interview schedule and consensus ratings. Moreover, conducting clinician ratings for every patient could be impossible in our busy daily clinical settings.

Among the self-report scales, versions of the Beck Depression Inventory (BDI, BDI-II) [10], Center for Epidemiologic Studies Depression Scale (CESD) [11], PHQ-9 [12] and versions of the Geriatric Depression Scale (GDS [13] and GDS-15 [14]) are popular among clinicians and clinical researchers. The BDI-II, CESD-revised (R), and PHQ-9 are based on DSM-IV criteria. Self-reporting of symptoms might be limited by recall bias and other personal reporting biases. However, standardized selfreporting scales generally show moderate or high correlation with clinician rating scales and can discriminate depression with appropriate sensitivity and specificity.

One of the strengths of the self-reporting scales is their potential for use in primary care settings and community 
settings without the need for rater education. It usually takes less than 10 minutes to complete these self-reporting forms.

Ms C had a score of 22 on the PHQ-9 scale when she visited the outpatient clinic. The PHQ-9 is composed of nine items derived from the Primary Care Evaluation of Mental Disorders screening questionnaire [15]. One or two minutes is enough to complete the scale, and it is suitable for screening and diagnosis of depression and for measuring treatment effects [12]. The PHQ-9 is also useful in the geriatric population [16].

Symptom changes can be measured in the clinic using a brief, easy-to-complete self-reporting scale (e.g. PHQ-9) along with other related symptom scales, and such a measurement may help people with depression in achieving remission. The State-Trait Anxiety Inventory or BAI could be useful for measuring symptoms of anxiety in a depression clinic. Measurement of physical symptoms (e.g. using the PHQ-15) is also helpful for achieving remission and improving patients' quality of life [17].

At her initial visit, Ms C was asked to complete a few self-reporting scales, such as the PHQ-9 (depression), BAI (anxiety) and PHQ-15 (somatization). During the interview, she was asked about adherence to her treatments. Her psychiatrist completed a Clinical Global Impressions Scale during every clinic visit.

When Ms C was reassessed after 6 weeks, her PHQ-9 total score decreased to 8 , which clinically implies a state of mild depression. However, the PHQ-15 still showed many somatic symptoms, such as fatigue, extremity numbness and sexual dysfunction. Her high BAI total score (24) still showed moderate anxiety. Her psychiatrist and family reported that she was much improved, but she still exhibited prominent residual symptoms.

\section{After 6 Weeks of Treatment, Ms C was Prescribed Aripiprazole to Control Residual Physical Symptoms and Anxiety. Do You Think that Augmentation will Work Better than Combination Therapy or Switching to Another Strategy in this Situation? (Drs Han, Pae, and Kato)}

After 6 weeks of treatment, aripiprazole ( $2.5 \mathrm{mg}$ daily) was added to Ms C's medication. Aripiprazole was increased to $10 \mathrm{mg}$ during the following 2 weeks, and depression symptoms were measured again at 12 weeks of treatment. The PHQ-9 total score decreased further to 2, which indicates remission, and zolpidem was removed from her medication list. Minimal dizziness and shortness of breath were still reported. Mild hand tremor was reported after the prescription of aripiprazole $(5 \mathrm{mg})$. However, Ms C reported general satisfaction with her depression treatment.
An augmentation strategy can be more efficient when the initially prescribed antidepressant (escitalopram in this case vignette) results in a response. Augmentation with aripiprazole at 6 weeks of treatment maintained partial responses to the previous antidepressant and helped the patient to achieve remission. Augmentation of antidepressant treatment can increase the risk of side effects and drug-drug interactions, and the cost of depression treatments, while switching strategies may increase the risks of loss of efficacy from the previous antidepressant [18].

Aripiprazole augmentation might be initiated much earlier than the time that this case was given it because patients with early response in an earlier treatment phase may have a better treatment outcome than those without early response in an earlier treatment phase [3, 19-21]. However, the conventional concept for adequate antidepressant treatment is 'wait and see' at least for 6-12 weeks before moving on to the next treatment strategy. Furthermore, there are no definite data or consensus about the most appropriate time for commencing the augmentation agent to current antidepressant yet [22].

\section{When is the Best Time to Start Augmentation or Combination Therapy? (Dr Chang)}

There is little evidence regarding the appropriate timing of augmentation or combination therapy in the treatment of depression. However, the existence of residual symptoms or lack of remission after 6-12 weeks of treatment with first-line antidepressants could justify second-line options, such as augmentation or combination therapy [22]. The STAR*D findings suggest that remission rates decrease considerably after two failed trials, supporting the recommendation that two failed trials defines treatment resistance. In STAR*D, almost $50 \%$ of the patients achieved remission during weeks 6-12 [4]. In the present case, aripiprazole was given after 6 weeks of antidepressant treatment. It appears that the treating psychiatrist may have considered that the patient presented at least moderate anxiety symptoms and also had a history of a chronic course.

\section{How Long Do You Continue Augmentation Drugs? (Dr Han)}

Evidence of long-term hazards of aripiprazole augmentation in depression patients is still lacking, although an open-label 52-week clinical trial showed long-term tolerability of the drug in patients with depression [23, 24]. For clinicians who treat patients with depression, the risk of tardive dyskinesia is important when prescribing AAPs, 
even though the risk is low with some AAPs such as aripiprazole [25]. Some clinicians adhere to a maximum of 6 months' use of AAPs, and others say 1 year. Currently, the most conservative way to use AAPs for depression is to taper the dose just after remission is achieved. Relapse and recurrence prevention studies should answer our clinical questions about adequate trial duration of AAPs for patients with depression.

\section{Can We Use Atypical Antipsychotic Augmentation for Patients with Depression Regardless of Chronicity or Recurrence of Depression? (Dr Pae)}

According to a 15-year prospective follow-up study of patients who recovered from an index episode of depression, a significant proportion of patients who experience one depressive episode are likely to experience another. In that study, $85 \%$ of the enrolled subjects $(n=380)$ experienced a recurrence, indicating the frequency of recurrence of depression in its natural clinical course [26]. The STAR $* D$ trial did not include AAPs as an option for next treatment steps. However, although the investigators did not intend to include chronic or recurrent MDD, almost $85 \%$ of subjects had such conditions [4, 27]. According to a recent subanalysis of the $\mathrm{STAR} * \mathrm{D}$ trial, remission rates were lowest and slowest for those with chronic index episodes [27]. Relapse was most likely for the chronic and recurrent group, whereas it was least likely for the nonchronic, nonrecurrent group [27]. A recent study of combined treatment of aripiprazole and venlafaxine at the beginning of treatment (12 weeks) found that the venlafaxine and aripiprazole combination was tolerable and efficacious in the treatment of patients with chronic or recurrent MDD, in which $70 \%$ of the subjects experienced remission at any time during the study [28]. Therefore, we may expect that aripiprazole augmentation with antidepressants should be another treatment option for chronic or recurrent depression in clinical practice; however, existing data are still limited.

\section{Is There Gender Effect in the Use of Atypical Antipsychotic Augmentation? (Dr. Pae)}

Published pooled data from two pivotal trials [29] indicated no specific clinical factors associated with the augmentation effect of aripiprazole for treating depression, with the exception of a gender interaction effect, which favoured female patients. However, the interaction effect was not qualitative in nature, as greater reductions in the MADRS total score were observed in the aripiprazole group than in the placebo group for both genders. Although overall greater treatment differences in favour of aripiprazole were observed in women than in men, the statistical significance of the treatment by gender effect in the combined analysis was primarily due to the results of the female portion in one pivotal trial result [30], two subsequent trials [31, 32] showed similar results between men and women for the primary efficacy measure. Therefore, we have no conclusive remarks on the role of the gender effect in aripiprazole augmentation for the treatment of depression.

\section{Summary}

Treatment of chronic depression with residual symptoms requires careful monitoring of care since residual depressive symptoms may negatively affect on the clinical course and outcomes in such patients. Timely evaluation and proper management at certain decision-making point by measurement-based care along with diverse treatment approaches are increasingly advocated in depression and such treatment strategy will eventually enhance response and remission rates in patients with chronic depression.

Acknowledgments Although Korea Otsuka International Asia and Arab (KOIAA) was involved in supporting the production of the supplement, the content of the manuscript, its review and revision, and the decision to submit to CNS Drugs were made solely by the authors and the supplement guest editor.

Sources of Financial Support The authors did not receive honorarium for writing this manuscript.

Conflict of interest Dr Han has received research grants from GlaxoSmithKline Korea, Eisai Korea, Korea Otsuka International Asia and Arab, Hanlim Pharmaceutical, Janssen Pharmaceuticals Korea, Eli Lilly and Company Korea, Korean Health Technology R\&D Project, Ministry of Health \& Welfare, Republic of Korea, Korean Research Foundation, and Otsuka Korea; and has received honoraria from GlaxoSmithKline Korea, Lundbeck Korea, AstraZeneca Korea, Janssen Pharmaceuticals Korea, Eisai Korea, Abott Korea, Eli Lilly and Company Korea, Norvatis Korea and Otsuka Korea. Dr Pae has received research grants from Eisai Korea, Janssen Pharmaceuticals Korea, Korean Health Technology R\&D Project, Ministry of Health \& Welfare, Republic of Korea, and Korean Research Foundation; and has received honoraria from Pfizer Korea and Otsuka Korea. Drs Yeh, Kato, Sato and Chang have received honoraria from Korea Otsuka International Asia and Arab.

Disclosure This manuscript has been published in a journal supplement that was created with an unrestricted educational grant from Korea Otsuka International Asia and Arab (KOIAA).

\section{References}

1. Moller HJ. Outcomes in major depressive disorder: the evolving concept of remission and its implications for treatment. World $\mathrm{J}$ Biol Psychiatry. 2008;9(2):102-14. 
2. Nierenberg AA, Husain MM, Trivedi MH, Fava M, Warden D, Wisniewski SR, et al. Residual symptoms after remission of major depressive disorder with citalopram and risk of relapse: a STAR*D report. Psychol Med. 2009;22:1-10.

3. Nierenberg AA, Keefe BR, Leslie VC, Alpert JE, Pava JA, Worthington JJ III, et al. Residual symptoms in depressed patients who respond acutely to fluoxetine. J Clin Psychiatry. 1999;60(4):221-5.

4. Rush AJ, Trivedi MH, Wisniewski SR, Nierenberg AA, Stewart JW, Warden D, et al. Acute and longer-term outcomes in depressed outpatients requiring one or several treatment steps: a STAR*D report. Am J Psychiatry. 2006;163(11):1905-17.

5. American Psychiatric Association. Diagnostic and statistical manual of mental disorders. 4th ed. Washington, DC: American Psychiatric Association; 2000.

6. Trivedi MH, Daly EJ. Measurement-based care for refractory depression: a clinical decision support model for clinical research and practice. Drug Alcohol Depend. 2007;88(Suppl. 2):S61-71.

7. Fava M, Rush AJ, Trivedi MH, Nierenberg AA, Thase ME, Sackeim HA, et al. Background and rationale for the sequenced treatment alternatives to relieve depression (STAR*D) study. Psychiatr Clin North Am. 2003;26(2):457-94; x.

8. Hamilton M. Development of a rating scale for primary depressive illness. Br J Soc Clin Psychol. 1967;6(4):278-96.

9. Carmody TJ, Rush AJ, Bernstein I, Warden D, Brannan S, Burnham D, et al. The Montgomery Asberg and the Hamilton ratings of depression: a comparison of measures. Eur Neuropsychopharmacol. 2006;16(8):601-11.

10. Beck ATSR, Brown GK. BDI-II beck depression inventory, 2nd edn manual. San Antonia: The Psychological Corporation; 1996.

11. Radloff LS. The CES-D scale: a self-report depression scale for research in the general population. Appl Psychol Meas. 1977; 1(3):385-401.

12. Kroenke K, Spitzer RL, Williams JB. The PHQ-9: validity of a brief depression severity measure. J Gen Intern Med. 2001;16(9): 606-13.

13. Yesavage JA. Geriatric depression scale. Psychopharmacol Bull. 1988;24(4):709-11.

14. Mitchell AJ, Bird V, Rizzo M, Meader N. Diagnostic validity and added value of the Geriatric Depression Scale for depression in primary care: a meta-analysis of GDS30 and GDS15. J Affect Disord. 2010;125(1-3):10-7.

15. Spitzer RL, Williams JB, Kroenke K, Hornyak R, McMurray J. Validity and utility of the PRIME-MD patient health questionnaire in assessment of 3000 obstetric-gynecologic patients: the PRIME-MD Patient Health Questionnaire Obstetrics-Gynecology Study. Am J Obstet Gynecol. 2000;183(3):759-69.

16. Han C, Jo SA, Kwak JH, Pae CU, Steffens D, Jo I, et al. Validation of the Patient Health Questionnaire-9 Korean version in the elderly population: the Ansan Geriatric Study. Compr Psychiatry. 2008;49(2):218-23.

17. Han C, Pae CU, Patkar AA, Masand PS, Kim KW, Joe SH, et al. Psychometric properties of the Patient Health Questionnaire-15 (PHQ-15) for measuring the somatic symptoms of psychiatric outpatients. Psychosomatics. 2009;50(6):580-5.

18. Connolly KR, Thase ME. If at first you don't succeed: a review of the evidence for antidepressant augmentation, combination and switching strategies. Drugs. 2011;71(1):43-64.

19. Kemp DE, Ganocy SJ, Brecher M, Carlson BX, Edwards S, Eudicone JM, et al. Clinical value of early partial symptomatic improvement in the prediction of response and remission during short-term treatment trials in 3369 subjects with bipolar I or II depression. J Affect Disord. 2011;130(1-2):171-9.

20. Szegedi A, Jansen WT, van Willigenburg AP, van der Meulen E, Stassen HH, Thase ME. Early improvement in the first 2 weeks as a predictor of treatment outcome in patients with major depressive disorder: a meta-analysis including 6562 patients. J Clin Psychiatry. 2009;70(3):344-53.

21. Szegedi A, Muller MJ, Anghelescu I, Klawe C, Kohnen R, Benkert O. Early improvement under mirtazapine and paroxetine predicts later stable response and remission with high sensitivity in patients with major depression. J Clin Psychiatry. 2003;64(4): 413-20.

22. Papakostas GI, Petersen TJ, Green C, Iosifescu DV, Yeung AS, Nierenberg AA, et al. A description of next-step switching versus augmentation practices for outpatients with treatment-resistant major depressive disorder enrolled in an academic specialty clinic. Ann Clin Psychiatry. 2005;17(3):161-5.

23. Berman RM, Thase ME, Trivedi MH, Hazel JA, Marler SV, McQuade RD, et al. Long-term safety and tolerability of openlabel aripiprazole augmentation of antidepressant therapy in major depressive disorder. Neuropsychiatr Dis Treat. 2011;7: 303-12.

24. Pae CU, Forbes A, Patkar AA. Aripiprazole as adjunctive therapy for patients with major depressive disorder: overview and implications of clinical trial data. CNS Drugs. 2011;25(2): 109-27.

25. Pae CU. A review of the safety and tolerability of aripiprazole. Expert Opin Drug Saf. 2009;8(3):373-86.

26. Mueller TI, Leon AC, Keller MB, Solomon DA, Endicott J, Coryell W, et al. Recurrence after recovery from major depressive disorder during 15 years of observational follow-up. Am J Psychiatry. 1999;156(7):1000-6.

27. Rush AJ, Wisniewski SR, Zisook S, Fava M, Sung SC, Haley CL, et al. Is prior course of illness relevant to acute or longer-term outcomes in depressed out-patients? A STAR*D report. Psychol Med. 2012;42(6):1131-49.

28. Nierenberg A, Trivedi M, Gaynes B, Mitchell J, Davis L, Husain $\mathrm{M}$, et al. Effectiveness study of venlafaxine-XR combined with aripiprazole for chronic or recurrent major depressive disorder. Aust N Z J Psychiatry. 2009;43:956-67.

29. Thase ME, Trivedi MH, Nelson JC, Fava M, Swanink R, Tran $\mathrm{QV}$, et al. Examining the efficacy of adjunctive aripiprazole in major depressive disorder: a pooled analysis of 2 studies. Prim Care Companion J Clin Psychiatry. 2008;10(6):440-7.

30. Berman RM, Marcus RN, Swanink R, McQuade RD, Carson WH, Corey-Lisle PK, et al. The efficacy and safety of aripiprazole as adjunctive therapy in major depressive disorder: a multicenter, randomized, double-blind, placebo-controlled study. J Clin Psychiatry. 2007;68(6):843-53.

31. Berman RM, Fava M, Thase ME, Trivedi MH, Swanink R, McQuade RD, et al. Aripiprazole augmentation in major depressive disorder: a double-blind, placebo-controlled study in patients with inadequate response to antidepressants. CNS Spectr. 2009;14(4):197-206.

32. Marcus RN, McQuade RD, Carson WH, Hennicken D, Fava M, Simon JS, et al. The efficacy and safety of aripiprazole as adjunctive therapy in major depressive disorder: a second multicenter, randomized, double-blind, placebo-controlled study. J Clin Psychopharmacol. 2008;28(2):156-65. 